\title{
Highly Efficient Cladding Pumped Dual-Wavelength Thulium Ytterbium Co-Doped Fiber Laser
}

\author{
A.A. LatifF ${ }^{a}$, I.M. Babar ${ }^{b}$, H. Shamsudin ${ }^{a}$, M.C. Paul $^{c}$, A. Halder $^{c}$, S. Das $^{c}$, \\ S.K. BhADRA ${ }^{c}$, H. AhMAD ${ }^{a}$ AND S.W. HARUN ${ }^{a, b, *}$ \\ ${ }^{a}$ Photonics Research Centre, University of Malaya, 50603 Kuala Lumpur, Malaysia \\ ${ }^{b}$ Department of Electrical Engineering, University of Malaya, 50603 Kuala Lumpur, Malaysia \\ ${ }^{c}$ Fiber Optics and Photonics Division, Central Glass and Ceramic Research Institute, CSIR, Kolkata, India \\ (Received June 21, 2016; in final form December 2, 2016) \\ This paper describes the all-fiber dual-wavelength thulium ytterbium co-doped fiber laser by using a newly \\ developed octagonal shaped double-clad thulium-ytterbium co-doped fiber as gain medium in conjunction with \\ spatial filtering effect. The thulium ytterbium co-doped fiber laser operates at wavelengths of $1990.64 \mathrm{~nm}$ and \\ $1998.92 \mathrm{~nm}$ with a signal to noise ratio of more than $34 \mathrm{~dB}$ as the $980 \mathrm{~nm}$ multimode pump power is increased \\ above a threshold value of $1.5 \mathrm{~W}$. The slope efficiency of the dual-wavelength laser is measured to be around \\ $26.16 \%$, which is comparable with the conventional thulium laser pumped by $800 \mathrm{~nm}$ single mode pump.
}

DOI: 10.12693/APhysPolA.130.1332

PACS/topics: $42.55 . \mathrm{Wd}, 42.60 . \mathrm{Fc}, 42.70 .-\mathrm{a}$

\section{Introduction}

Fiber lasers operating in $2 \mu \mathrm{m}$ region have attracted great research interest in recent years because of their potential scientific applications in various fields such as in remote sensing, spectroscopy and biomedical application $[1,2]$. The wavelength also overlaps with many absorption lines of several gas molecules such as hydrogen bromide $(\mathrm{HBr})$ and carbon dioxide $\left(\mathrm{CO}_{2}\right)$ [3], which creates the possibility of constructing cost-effective gas sensors. Strong water absorption in this wavelength range makes the light source extremely desirable in biomedical applications. It has been shown that $2 \mu \mathrm{m}$ laser outperform $1.0 \mu \mathrm{m}$ and $1.5 \mu \mathrm{m}$ sources in dermatology and surgery, serving as precise and efficient optical scalpels $[4,5]$. The $2 \mu \mathrm{m}$ fiber laser can be realized using thulium-doped fiber (TDF) as the gain medium in conjunction with $794 \mathrm{~nm}$ pumping. However, the lasing threshold for achieving an efficient ${ }^{3} F_{4}$ and ${ }^{3} H_{6}$ transition is relatively high and a power of the available commercial single mode $794 \mathrm{~nm}$ laser diode is limited to around $180 \mathrm{~mW}$.

Of late, a thulium ytterbium co-doped fiber laser (TYDFL) was also demonstrated for oscillation around $2 \mu \mathrm{m}$ based on ytterbium ( $\mathrm{Yb}$ ) to thulium (Tm) energy transfer [6]. It uses a commercially available and cheaper laser diode operating within 905 to $980 \mathrm{~nm}$ as a pump source. This is due to the ${ }^{3} \mathrm{H}_{5}$ level of $\mathrm{Tm}^{3+}$, which is (quasi-) resonant with the excited $\mathrm{Yb}^{3+}$ level $\left({ }^{2} F_{5 / 2}\right)$ and thus allows the energy transfer to create a population inversion in $\mathrm{Tm}^{3+}[7,8]$. In the previous work, Jeong et al. demonstrated a TYDF operating in D-shaped geometry multimode with efficiency of around $32 \%$ [9]. To find the

*corresponding author; e-mail: swharun@um.edu.my best laser performance which is appropriate to specific applications, various $2 \mu \mathrm{m}$ fiber laser configurations have developed. Dual-wavelength running fiber lasers is one of the laser that has been under intensive research recently due to its wide application potential in ranging, communication, microwave photonics, terahertz generation, and optical instrument testing $[10,11]$. Up to date, there were many techniques have been proposed and demonstrated to achieve a dual-wavelength lasing such as by employing a dual-cavity configuration and twin-peak reflection grating. For instance, Zhou et al. [12] demonstrated a room temperature all-fiber dual-wavelength TDFL based on cascaded fiber Bragg grating (FBG) array. However, the FBG structure requires an expensive UV laser to be fabricated and the conventional FBG cannot operate at temperature higher than $300^{\circ} \mathrm{C}$.

In this paper, a room temperature all-fiber dualwavelength TYDFL operating in $2 \mu \mathrm{m}$ region is demonstrated for the first time using a newly developed an orthogonal shaped double-clad thulium-ytterbium codoped fiber (TYDF) as a gain medium in a half-opened linear laser cavity. In this cavity, one fiber end is connected to a reflector whereas the other end is open. In this work, a high-reflectivity optical mirror is used as a reflector to reduce the threshold pump power while a $980 \mathrm{~nm}$ multimode laser diode is used to pump the TYDF via a multimode combiner. The dual-wavelength operation is realized in the proposed laser based on spatial filter generated due to mismatch at the splicing points of TYDF.

\section{TYDF fabrication and their working principle}

In this work, a proposed double-clad octagonal shaped TYDF, which was coated by a low refractive index polymer, was used as the gain medium. It was drawn by a preform, which was fabricated by the modified chemical vapor deposition (MCVD) process in conjunction with 


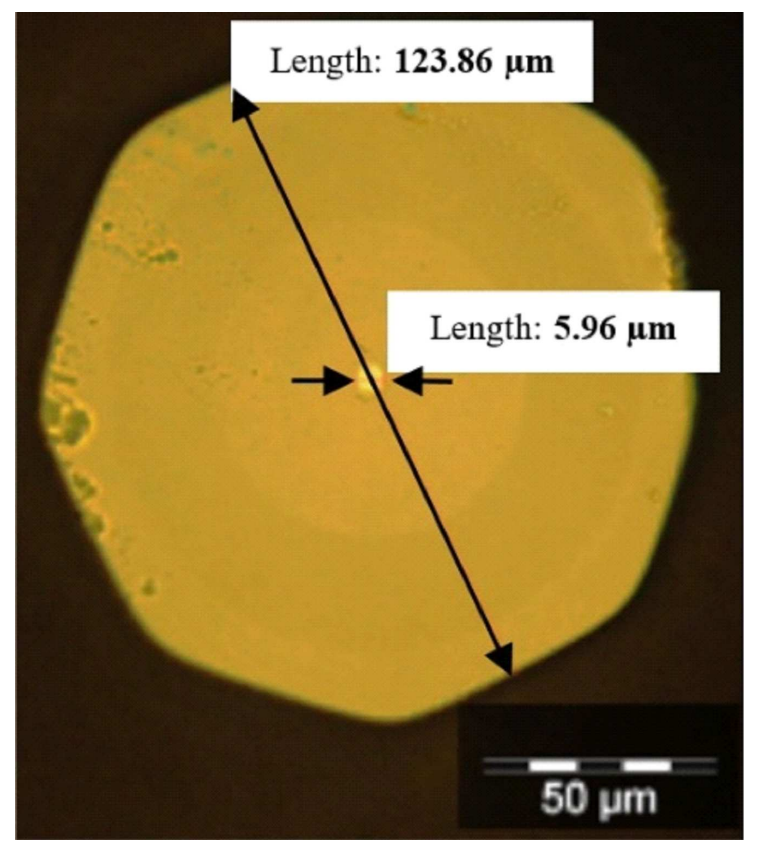

Fig. 1. The microscopic cross-sectional view of an octagonal shaped double-clad fiber used as the gain medium.

the solution doping technique. Figure 1 shows the microscopic view of fiber cross-section, which indicates our newly developed double-clad structure with a core and inner-cladding diameters of $5.96 \mu \mathrm{m}$ and $123.86 \mu \mathrm{m}$, respectively. The numerical aperture (NA) of the fabricated TYDF core is measured to be 0.23

The preform was made by deposition of two porous unsintered $\mathrm{SiO}_{2}$ soot layers inside a pure silica glass tube at temperature around $1550 \pm 10^{\circ} \mathrm{C}$. The porous layers were soaked by an alcoholic solution containing doping elements i.e. $\mathrm{Tm}, \mathrm{Yb}, \mathrm{Y}$, and $\mathrm{Al}$ for about $30 \mathrm{~min}$ to achieve efficient doping. Then, dehydration and oxidation were performed at the temperature around 900 to $1000^{\circ} \mathrm{C}$. Sintering of the un-sintered layers was also done by slow increase of the temperature from 1500 to $2000^{\circ} \mathrm{C}$ using the conventional MCVD technique. Upon completion of sintering as well as oxidation, the tube was slowly collapsed to convert it into optical preform. Based on electron probe micro analyser (EPMA) result, we found out that the fabricated optical preform consists of $\mathrm{Al}_{2} \mathrm{O}_{3}$, $\mathrm{Y}_{2} \mathrm{O}_{3}, \mathrm{Tm}_{2} \mathrm{O}_{3}$ and $\mathrm{Yb}_{2} \mathrm{O}_{3}$ dopants with average weight percentage of $5.5,3.3,0.7$, and $4.0 \mathrm{wt} \%$, respectively. The composition of dopants in this fiber was optimized to allow the formation of smaller phase-separated nanoparticles of $3-5 \mathrm{~nm}$, which functions to reduce the background loss of the fiber as well as to increase the energy transfer efficiency from $\mathrm{Yb}$ to $\mathrm{Tm}$ ions. The presence of $\mathrm{Al}_{2} \mathrm{O}_{3}$ and $\mathrm{Y}_{2} \mathrm{O}_{3}$ helps to decrease the phonon energy of alumino-silica glass, which assists in preventing the clustering of $\mathrm{Yb}$ and $\mathrm{Tm}$ ions into the core glass matrix and thus increases the probability of radiative emission.

The fabricated circular preform is converted to octagonal shape through grinding followed by polishing method.
The geometrically modified preform is then drawn at temperature of $2050{ }^{\circ} \mathrm{C}$ to obtain an octagonally shaped fiber with outer cladding diameter of $125 \mu \mathrm{m}$. The fiber is then coated with a low refractive index polymer to ensure the robustness of the fiber. The doping levels of $\mathrm{Tm}^{3+}$ and $\mathrm{Yb}^{3+}$ ions in the fabricated TYDF are $4.85 \times 10^{19} \mathrm{ions} / \mathrm{cc}$ and $27.3 \times 10^{19} \mathrm{ions} / \mathrm{cc}$, respectively. The $\mathrm{Tm}^{3+}$ and $\mathrm{Yb}^{3+}$ cladding absorptions of the fiber are measured to be around 0.325 and $3.3 \mathrm{~dB} / \mathrm{m}$ at $790 \mathrm{~nm}$ and $976 \mathrm{~nm}$, respectively.

As opposed to the conventional single-clad fiber, where the pump light is coupled directly into the core, the pump light travels down the fiber in the inner cladding for the double-clad fiber. When the pump light overlaps with the core, it gets absorbed by the dopants. Such octagonal geometry of the cladding improves the pump absorption efficiency. Octagonal geometrical shape has more broken circular geometry (eight sides) and symmetrical crosssection compared to the conventional hexagonal shape. The higher number of broken circular geometry could provide more pump absorption, which in turn increases the laser output power [13]. In addition, more symmetrical cross-section in the octagonal geometry could provide a lower splicing loss.

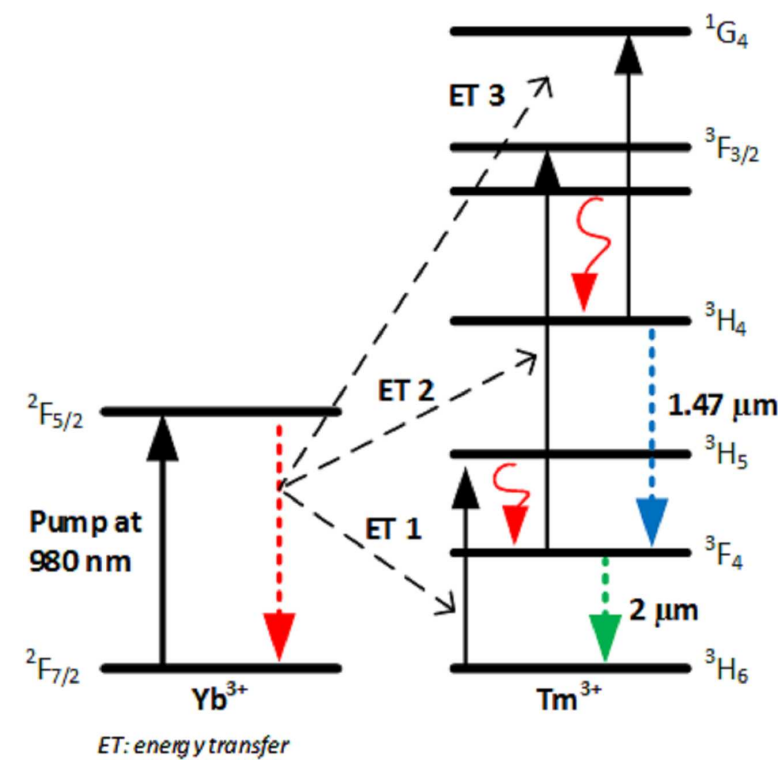

Fig. 2. Energy diagram levels for both ytterbium and thulium ions in TYDF showing an energy transfer.

The TYDFL operates based on the energy transition as shown in Fig. 2. Since, the active fiber has ytterbium ions as sensitizer, it employs up-conversion process to generate laser at $2 \mu \mathrm{m}$ region. The pumping was carried out using a $980 \mathrm{~nm}$ multimode laser diode, which is available in high power, easily obtained and relatively cheaper compared to other wavelength range. The pump propagates inside inner cladding of the fiber and the octagonally shaped fiber is expected to increase the overlapping factor of the pump and ions. As the pump photons are absorbed by the ytterbium ions, it excites the ions 
from ground state ${ }^{2} F_{7 / 2}$ to ${ }^{2} F_{5 / 2}$. As the ion relaxes to ground state, energy transfer process happens to neighboring thulium ion. When the thulium ions in ground state $\left({ }^{3} \mathrm{H}_{6}\right)$ absorb the donated photons it got elevated to ${ }^{3} \mathrm{H}_{5}$ level before it irradiatively relaxes to ${ }^{3} F_{4}$. Thulium ions that populate this state level drops to ground state again generating the $2 \mu \mathrm{m}$ laser.

\section{TYDFL experimental configuration}

Figure 3 shows the experimental setup for dualwavelength TYDFL with a half-opened linear cavity configuration, which is formed by the broadband mirror and an output coupler reflector. A $15 \mathrm{~m}$ long homemade TYDF, which is pumped by a $980 \mathrm{~nm}$ multimode laser diode ( $\mathrm{LD}$ ) via a multimode combiner (MMC), is used as the gain medium. The $15 \mathrm{~m}$ is the optimized length for the available pump power. The output coupler reflector is formed by the $4 \%$ Fresnel reflection from the perpendicular cleaved fiber end. The laser output is measured by using an optical spectrum analyzer (OSA) with a resolution of $0.02 \mathrm{~nm}$ and an optical power meter (OPM).

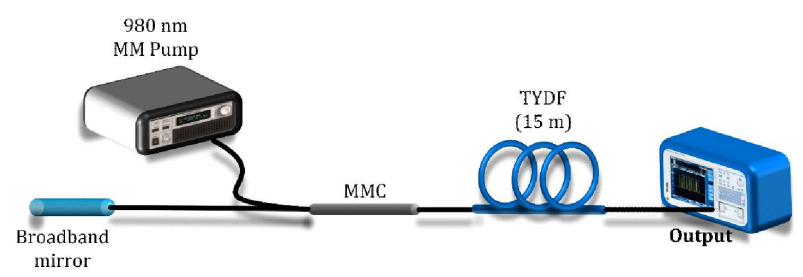

Fig. 3. Experimental setup of the proposed dual wavelength TYDFL.

\section{Results and discussions}

The laser operation is supposed to be grounded on the TYDF gain and the resonance between broadband mirror and a $4 \%$ Fresnel reflection from the perpendicular cleaved fiber end. When the TYDF is pumped by $980 \mathrm{~nm}$ laser to generate population inversion of ytterbium ions, the emitted energy is then transferred to thulium ions to generate an amplified spontaneous emission (ASE) in $2 \mu \mathrm{m}$ region via spontaneous and stimulated emission process. The ASE oscillates in the linear cavity to generate laser when the gain overcomes the total cavity loss. With increase of power of the pump, dual-wavelength laser is generated due to the effect of the phase mismatch at the splicing points of a TYDF birefringence.

As the geometry of TYDF is significantly different with both fibers spliced to it, the power oscillations occur within the TYDF, thus producing some wavelengthdependent losses. As a results of specific transmission function of the spatial mode-beating filter, the signals at two different wavelengths can oscillate in the cavity whenever the difference of the cavity losses for them are somewhat reduced. At sufficient pump power, the cavity losses for at least two wavelength reduced to seek dualwavelength oscillations with the aid of the TYDF, whose gain broadening behaves inhomogeneously.

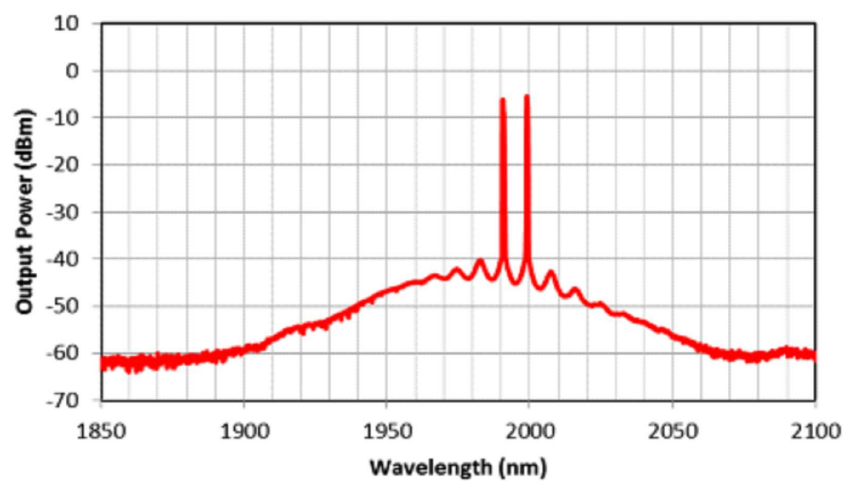

Fig. 4. Output spectrum of the proposed dualwavelength TYDFL at multimode pump power of $1.5 \mathrm{~W}$.

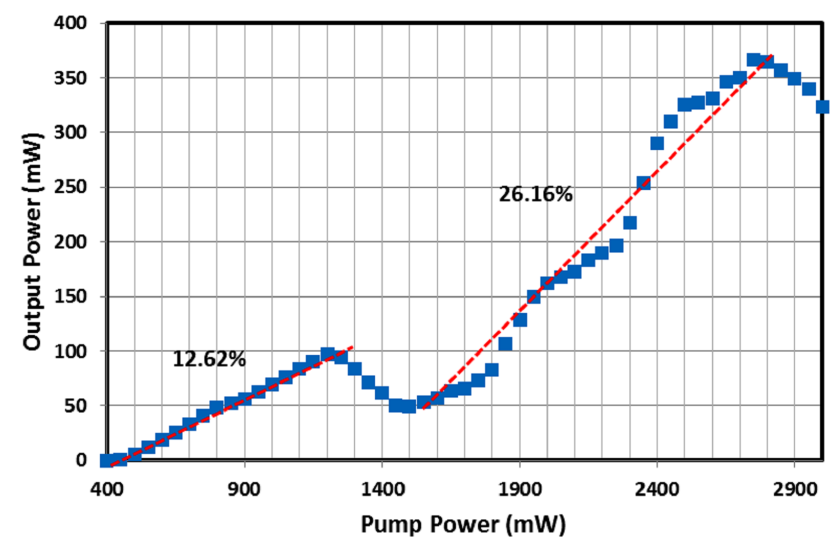

Fig. 5. Output power characteristic against the pump power.

The output spectrum of the proposed laser as recorded by an OSA is shown in Fig. 4 when the multimode $980 \mathrm{~nm}$ pump is fixed at $1.5 \mathrm{~W}$, respectively. As seen in the figure, a dual-wavelength output lines are obtained at 1990.64 and $1998.92 \mathrm{~nm}$ with a signal to noise ratio of more than $34 \mathrm{~dB}$. The $3 \mathrm{~dB}$ bandwidth of both lasers is measured to be less than $0.2 \mathrm{~nm}$. The power difference between the two peaks is less than $1 \mathrm{~dB}$. The relation between the output powers of the dual-wavelength laser against the multimode pump power is shown in Fig. 5. The TYDFL starts to lase at pump power of $400 \mathrm{~mW}$ to generate a random laser operating at around $1995 \mathrm{~nm}$ region. The output power of the laser is observed to increase linearly with the increment of multimode pump power up to pump power of $1200 \mathrm{~mW}$ with a slope efficiency of $12.62 \%$. The output power suddenly drops at pump power above $1200 \mathrm{~mW}$ to enable dual-wavelength laser generation.

The dual-wavelength laser starts to lase at threshold power of $1550 \mathrm{~mW}$ before both output peaks power increases linearly with the increment of multimode pump power up to pump power of $2750 \mathrm{~mW}$. The slope efficiency of the dual-wavelength laser is measured to be around $26.16 \%$, which is comparable with the conventional TDFL pumped by $800 \mathrm{~nm}$ single mode pump. 
For TYDFL system, this is the highest slope efficiency, which has been reported so far. At the multimode pump power of $2750 \mathrm{~mW}$, the dual-wavelength laser produces the maximum total output power of $366 \mathrm{~mW}$. As the pump power is increased above $2750 \mathrm{~mW}$, the total output power drops due to more number of lines are generated in the laser.

\section{Conclusion}

Let us summarize the main results of this report. An all-fiber dual-wavelength TYDFL operating at $2 \mu \mathrm{m}$ region is demonstrated based on spatial filtering effect. It uses a newly developed octagonal shaped double-clad TYDF as gain medium. The laser developed by us generates the dual-wavelength output lines at wavelengths of 1990.64 and $1998.92 \mathrm{~nm}$ as the $980 \mathrm{~nm}$ multimode pump power is increased above a threshold value of $1.5 \mathrm{~W}$. The signal to noise ratios of the both laser lines are measured to be more than $34 \mathrm{~dB}$ and the slope efficiency of the laser is $26.16 \%$. The use of chirped FBG as a reflector may enhance the laser performance.

\section{References}

[1] Y. Zhang, Y. Tian, W. Wang, B. Yao, Laser Phys. Lett. 7, 225 (2010).

[2] P. Zhang, W. Ma, T. Wang, Q. Jia, C. Wan, Chin. Opt. Lett. 12, 111403 (2014).
[3] W. Zeller, L. Naehle, P. Fuchs, F. Gerschuetz, L. Hildebrandt, J. Koeth, Sensors (Switzerland) 10, $2492(2010)$.

[4] N.M. Fried, K.E. Murray, J. Endourol. 19, 25 (2005).

[5] R. Szlauer, R. Götschl, A. Razmaria, L. Paras, N.T. Schmeller, Eur. Urol. 55, 368 (2009).

[6] A. Halder, M.C. Paul, S.S.A. Damanhuri, N.A.D. Huri, A. Hamzah, S.W. Harun, H. Ahmad, S. Das, M. Pal, S.K. Bhadra, J. Mod. Opt. 59, 527 (2012).

[7] S.W. Harun, N. Saidin, S.S.A. Damanhuri, H. Ahmad, A. Halder, M.C. Paul, S. Das, M. Pal, S.K. Bhadra, Laser Phys. Lett. 9, 50 (2012).

[8] M.Y. Jeon, N. Kim, J. Shin, J.S. Jeong, S. Han, C.W. Lee, Y.A. Leem, D. Yee, H.S. Chun, K.H. Park, Opt. Expr. 18, 12291 (2010).

[9] Y. Jeong, P. Dupriez, J.K. Sahu, J. Nilsson, D. Shen, W.A. Clarkson, in: Proc. Europ. Symp. on Optics and Photonics for Defence and Security, 2004, p. 28.

[10] H. Ahmad, M.Z. Zulkifli, A.A. Latiff, S.W. Harun, Opt. Commun. 282, 4771 (2009).

[11] H. Li, H. Ding, K.T. Chan, Electron. Lett. 33, 52 (1997).

[12] P. Zhou, X.L. Wang, Y.X. Ma, K. Han, Z.J. Liu, Laser Phys. 21, 184 (2011).

[13] N. Saidin, M.S.Z. Abidin, S.S.A. Damanhuri, A. Haider, M.C. Paul, S.W. Harun, S. Das, M. Pal, S.K. Bhadra, in: Proc. IEEE 6th Int. Conf. on Photonics, 2016, p. 1. 